\title{
The spatiotemporal distinctiveness of direct causation
}

\author{
Michael E. Young and Steven Sutherland \\ Southern Illinois University, Carbondale, Illinois
}

\begin{abstract}
The launching effect, in which people judge one object to have caused another to immediately move after contact, is often described as the prototype of direct causation. The special status of this interaction may be due to its psychophysical distinctiveness, and this property may be the origin of the formation of causality as a conceptual category. This hypothesis was tested by having participants judge the relative similarity of pairs of events that either had no spatial gap or delay (direct launching) or had small gaps and/or short delays. Direct launching was much easier to discriminate from launches involving small gaps or delays. In a follow-up experiment, participants made similar judgments for a noncausal event.
\end{abstract}

The mystery of the psychological nature of causality has produced a resurgence of interest in recent years. Researchers have examined the development of causal understanding (Gopnik et al., 2004; Rochat, Striano, \& Morgan, 2004; Schlottmann, Allen, Linderoth, \& Hesketh, 2002), the acquisition of causally relevant information across multiple exposures (Allan \& Tangen, 2005; Buehner \& May, 2003; Castro \& Wasserman, 2007; Lagnado \& Sloman, 2006; Perales, Catena, Shanks, \& Gonzalez, 2005), reasoning from given information to sound causal claims (Fugelsang \& Thompson, 2003; Pearl, 2000; Tenenbaum, Griffiths, \& Kemp, 2006; Waldmann \& Hagmayer, 2005), and the automatic impression of causality from a single observation of interacting objects (Blakemore et al., 2001; Guski \& Troje, 2003; Scholl \& Nakayama, 2002; Young, Rogers, \& Beckmann, 2005). Each of these lines of research has revealed interesting aspects of the psychological nature of causality and uncovered discrepancies between the normatively correct process of judging causation and the process actually used by human judges.

The origin of causality as a concept is often tied to the simplest and most predictable of causal relationships extant in our world - the physical contact of interacting objects (Leslie \& Keeble, 1987; Michotte, 1946/1963; White, 1988). From infancy, we observe and participate in interactions in which one object contacts another object and causes it to move. Parents are observed to open doors, pick up bottles, and move a pacifier. Young children kick a mobile, grab a toy, or push objects off a table. These causeeffect interactions are immediate and straightforward, unlike the causal relationships between smoking and cancer or our diet and our health, both of which involve delayed and probabilistic relationships. It thus seems plausible that experience with direct causal interactions may provide the basis for more advanced causal learning and reasoning.
In the present project, we examined the psychophysical discrimination of direct causation from distal and delayed interactions. Instead of contrasting idealized causation (e.g., one billiard ball striking and launching another) with a variety of real-world examples of ambiguous, delayed, and distal causal or noncausal interactions (e.g., exercise producing weight loss, appearance affecting mating success, or driving skill altering accident likelihood), we chose the more modest goal of identifying the relative similarity of direct launching, distal interactions with small gaps, and delayed interactions with small delays. The effects of spatial and temporal contiguity on causal judgments involving launching have been extensively studied (e.g., Guski \& Troje, 2003; Leslie, 1984; Michotte, 1946/1963; Young et al., 2005), and researchers have demonstrated that judgments are systematically influenced by both delays and gaps, with direct launching (no delay, no gap) having generated distinctly higher causal judgments.

Although philosophers, scientists, and psychologists have exerted considerable effort identifying the strict conditions under which we should identify a relationship as causal, in everyday language causal terms are used much more loosely, without undue consideration of their correct usage. Given that we cannot actually see the causal forces in action, causality must be inferred from observed variables. One such variable, spatiotemporal contiguity, appears central to the identification of causality. In our musing on the issue, we began to consider the possibility that spatiotemporal contiguity might represent a distinct relational category in the world and, thus, have given rise to the relation receiving a unique label-causal - in the evolution of language. The distinctive nature of direct interactions may give rise to their special status, their rapid learning, and the historical development of special linguistic labels for this class of events. Surprisingly, no data

M. E.Young, meyoung@siu.edu 
exist in which the similarity of direct, delayed, and distal interactions was examined, so we sought to evaluate our hypothesis empirically.

A demonstration that spatiotemporal contiguity is distinctly different from other interactions, however, gives rise to an interesting chicken-and-egg problem: Is direct launching (à la Michotte) uniquely different from similar interactions that lack contiguity because of its spatiotemporal uniqueness or because of its causality? Spatiotemporal properties are easy to manipulate, but causality cannot be manipulated so directly. Might spatiotemporal contiguity be unique and, thus, have given rise to its receiving a special linguistic label, or might causality be unique and have given rise to the ability to easily discriminate spatiotemporally contiguous relations from noncontiguous relations?

In two experiments, we simply asked participants whether two animations were identical or different. The instructions included no mention of causality, and thus, the task required a simple perceptual discrimination (although in Experiment 2, some participants judged causality before making the discrimination). It was our suspicion that a direct causal interaction would be easier to discriminate from other types of interactions simply because it involved the endpoints of the delay and distance continua. As a control, our second experiment consisted of an interaction that was not causal to determine whether the uniqueness of spatiotemporal contiguity in a direct launch was a by-product of its causality, rather than of its spatial and temporal properties.

\section{EXPERIMENT 1}

\section{Method}

A pilot study involving 0 - to $200-\mathrm{msec}$ delays and 0 - to $1.2-\mathrm{cm}$ gaps revealed that observers found it very difficult to discriminate between events with such small delay differences; this result suggested a restricted range problem. For the present study, we increased the range of delay from 0 to $600 \mathrm{msec}$ to ensure clear discrimination of both dimensions.

Participants. A total of 21 introductory psychology students at Southern Illinois University at Carbondale received course credit for their voluntary participation.

Stimuli. The participants saw pairs of 16 different animations: 4 gaps $\left(0.0,0.3,0.6\right.$, or $1.2 \mathrm{~cm}$, or $0.00^{\circ}, 0.28^{\circ}, 0.57^{\circ}$, or $1.15^{\circ}$ of visual angle $) \times 4$ delays $(0,150,300$, or $600 \mathrm{msec})$. For the direct launching animation (no delay and no gap), the left object moved to the right and stopped when it was contiguous to the right object; after contact, the right object immediately began moving to the right. For the delay animations, the left object again moved to the right and stopped; the right object began moving to the right 150,300, or $600 \mathrm{msec}$ after the left object had stopped moving. For the gap animations, the left object moved to the right and stopped when its rightmost edge was the programmed distance from the leftmost edge of the right object, at which point the right object immediately began moving to the right. For those animations with both a delay and a gap, both contingencies were applied.

A single animation of one ball's movement was created using LightWave 3D, Version 6, and was saved as a QuickTime movie, using Sorenson video compression. An animation light source was placed to the upper left and front (toward the observer) of the moving objects to provide realistic shading. Each ball was $1.4 \mathrm{~cm}\left(1.3^{\circ}\right.$ of visual angle) in diameter and used LightWave's "Clothing.tga" texture to provide color; the objects were rendered against a black background. When moving, each object moved at a steady rate of $4.0 \mathrm{~cm} / \mathrm{sec}\left(3.8^{\circ} / \mathrm{sec}\right)$. For every animation, the launching object appeared from off screen on the left side.

Presentation of the animations and collection of response data was programmed using PsyScope, Version XB46. To create one of the 16 animation movie types, the program started and stopped two movies, one for each ball, placed side by side on the screen. The program changed the duration of delay between the animations' stopping and starting times and the relative position of the movies in order to create the chosen gap/delay combination. After the first animation (comprising two component animations) had been played, the program presented a $10-\mathrm{cm}\left(9.55^{\circ}\right)$ mask (cubic pieces of the balls were randomly spread across a $5 \times 1 \mathrm{~cm}$ rectangular region) for 1,200 msec in order to erase any visual aftereffect of the ending position of the two balls, followed by the second animation (also comprising two component animations).

Procedure. The participants were seated at one of four identically configured 1.25-GHz Mac Mini computers. The participants first heard general instructions and then read more specific instructions that included the following:

In this experiment, your task is relatively simple. You will observe pairs of object animations each involving two objects. After observing both, you are to press the " 1 " key if you believe the two animations were the same and the " 3 " key if they were different.

Note, you should pay attention to differences in the distance between the two balls and the length of any delay between the action of the first object and the second object.

Once each participant indicated an understanding of the procedure, the experimenter started each of the programs.

On each trial, a gap/delay combination was randomly selected to be displayed first, and another combination was randomly selected to be second. Because of the large number of possible animation pairings (a total of $16 \times 16$, or 256 , pairings), we presented only 100 randomly selected (without replacement) pairings. Because our earlier work revealed that participant attention was not maintained when we tried to present all 256 combinations, we chose to sample from the stimulus space.

Table 1

Maximum Likelihood Estimates (MLEs) and Standard Errors for the Gap $(G)$, Delay $(D)$, and Constant (C) Parameters of Equation 1

\begin{tabular}{|c|c|c|c|c|c|c|}
\hline \multirow{3}{*}{$\begin{array}{l}\text { Stimulus } \\
\text { Condition }\end{array}$} & \multicolumn{6}{|c|}{ Function Parameter } \\
\hline & \multicolumn{2}{|c|}{$G$} & \multicolumn{2}{|c|}{$D$} & \multicolumn{2}{|c|}{ C } \\
\hline & MLE & $S E$ & MLE & $S E$ & MLE & $S E$ \\
\hline
\end{tabular}

\begin{tabular}{lrrrrrr}
\hline \multicolumn{7}{c}{ Experiment 1: Direct Approach Discrimination } \\
Direct & & & & & \\
$\quad$ First & 14.3 & 3.1 & 19.1 & 7.0 & -4.2 & 1.8 \\
$\quad$ Second & 44.5 & 9.8 & 7.4 & 2.1 & -3.4 & 1.6 \\
Distal/delayed & & & & & & \\
$\quad$ First & 13.0 & 2.5 & 4.5 & 1.9 & -7.6 & 1.7 \\
$\quad$ Second & 3.5 & 0.7 & 1.5 & 0.6 & -1.3 & 0.5
\end{tabular}

Experiment 2: Indirect Approach Discrimination

\begin{tabular}{lllllll} 
Direct & \multicolumn{7}{c}{ First } & 9.1 & 2.1 & 3.9 & 1.4 & -2.7 & 0.9 \\
$\quad$ Second & 4.9 & 1.2 & 2.2 & 0.6 & -0.8 & 0.3 \\
Distal/delayed & & & & & & \\
$\quad$ First & 4.8 & 0.8 & 0.5 & 0.5 & -2.6 & 0.6 \\
$\quad$ Second & 4.7 & 1.0 & 1.6 & 0.6 & -1.6 & 0.7 \\
& \multicolumn{7}{c}{ Experiment 2 2: Ratings } & & & \\
$\quad$ Direct approach & 1.5 & 0.3 & 2.7 & 0.4 & -1.3 & 0.3 \\
Indirect approach & 0.1 & 0.3 & 1.6 & 0.3 & -0.4 & 0.2 \\
\hline
\end{tabular}



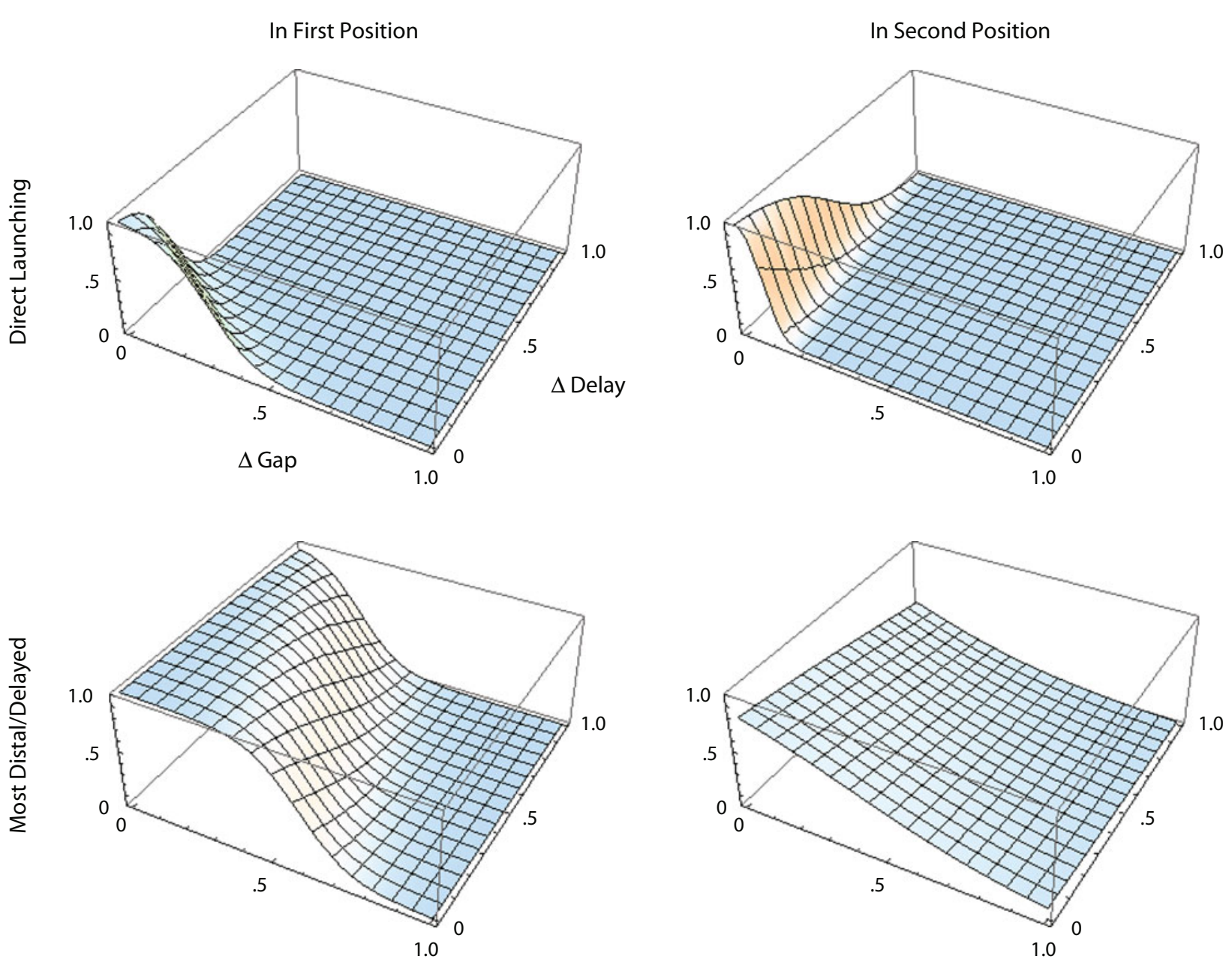

Figure 1. Experiment 1: Fitted empirical similarity to direct launching (top two figures) and the most distal/delayed interaction (bottom two figures) when the focal event was presented first (left two figures) or second (right two figures) in the comparison pair. The focal event of each analysis (direct launching in top two figures, distal/delayed in bottom two) is plotted at the $(0,0)$ coordinates. The gap and delay axes were rescaled to a standardized $0-1$ range; difference in gap is shown along the bottom left axis, difference in delay along the bottom right axis.

Due to the randomization procedure, not all gap and delay differences occurred an equal number of times. For example, a delay difference of $600 \mathrm{msec}$ could occur only when one of the movies had a 0 -msec delay and the other a $600-\mathrm{msec}$ delay, but a 0 -msec difference could occur for a $0 / 0,150 / 150,300 / 300$, or $600 / 600 \mathrm{msec}$ pairing. The experienced relative frequency of a particular combination of differences ranged from $1.1 \%$ for a difference of $0.3 \mathrm{~cm}$ and $450 \mathrm{msec}$ to $10.3 \%$ for a difference of $0.3 \mathrm{~cm}$ and $150 \mathrm{msec}$. Because of our stimulus sampling design, the use of repeated measures, and our desire to fit nonlinear models, we used nonlinear mixed effects modeling (nlme; Pinheiro \& Bates, 2004) to analyze the data.

Each trial comprised the presentation of the first animation, a 1,200 -msec duration mask, the presentation of the second animation, and a prompt, "Press ' 1 ' if the SAME, Press ' 3 ' if DIFFERENT." The intertrial interval was $500 \mathrm{msec}$.

\section{Results and Discussion}

All 21 participants successfully completed the task. The mean accuracy was $75 \%$ correct, ranging from $68 \%$ to $88 \%$ for individual participants. Our primary interest was the degree to which a particular stimulus, direct launch- ing, was distinctly discriminable from the other stimuli. As a baseline of comparison, we also considered the stimulus with the weakest spatiotemporal contiguity $(1.2-\mathrm{cm}$ gap, 600-msec delay) and its discriminability from the other stimuli. We chose this stimulus because the range of its difference scores $(0.0-1.2 \mathrm{~cm}$ and $0-600 \mathrm{msec})$ would match that of the direct launching. We performed a nonlinear mixed effects analysis using R's nlme function (assuming a diagonal covariance matrix) to identify the maximum likelihood estimates of the parameters of the following multidimensional sigmoid function:

$$
P(\mathrm{R}=\text { "same" })=\frac{1}{1+e^{G x+D y+L x y+C}},
$$

where $x$ is the difference in size of the gap $(0.0-1.2 \mathrm{~cm}$, rescaled to the $0-1$ range), $y$ is the difference in the size of the delay $(0-600$, rescaled to the $0-1$ range), $G$ is the degree of discriminative control for the gap, $D$ is the control by delay, $I$ is the strength of the interaction between gap and delay effects, and $C$ is the response bias. Nonlinear 
mixed effects analysis estimates parameter values using a multilevel analysis in which estimates across participants (the fixed effects) are a by-product of the estimates for each participant (the random effects), and each participant's estimates are influenced by the estimates derived from other participants. This process allows the nlme to estimate the full range of the function for each participant, even if a particular participant did not experience one or more stimulus combinations.

In each of our analyses, models that excluded the interaction term produced lower Bayesian information criterion values than did models that included the interaction, so the simpler main effects models were preferred. We performed four nlme analyses: when the direct launching was (1) first or (2) second and when the distal and delayed action was (3) first or (4) second. The first two analyses assess the participants' ability to discriminate the direct launching from launchings involving delays and/or gaps. The second two analyses assess the ability to discriminate the most distal and delayed launch from those with smaller gaps and/or delays. By examining the key event in both the initial and second positions, we were able to examine possible order effects and replicate any stimulus effects.

The maximum likelihood estimates are shown in Table 1, and the results are shown graphically in Figure 1. The nlme analysis revealed that the direct launching was judged to be distinctly different from the other stimuli. The discriminative control by space and time were approximately equal when the direct launching was first, but the gap had more control when the direct launching was second.

In contrast, the most distal and delayed interaction was frequently confused with the other distal and/or delayed interactions, especially when the comparison animation involved delay differences rather than gap differences. Control by gap was consistently stronger, especially so when the most distal and delayed interaction was the first animation viewed (in this case, control by delay did not reach statistical significance).
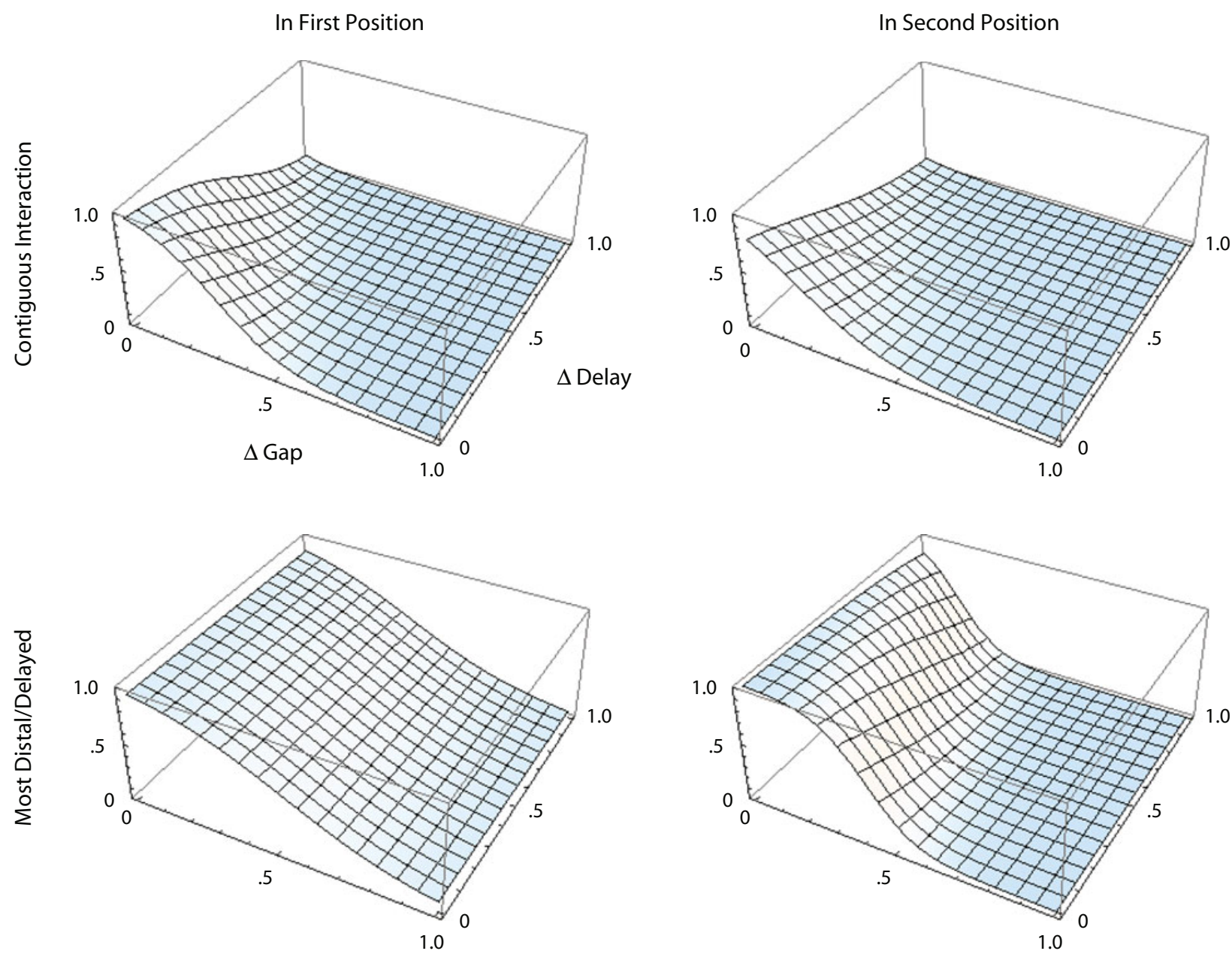

Figure 2. Experiment 2: Fitted empirical similarity to contiguous interaction (top two figures) and the most distal/delayed interaction (bottom two figures) when the focal event was presented first (left two figures) or second (right two figures) in the comparison pair. The focal event (contiguous interaction in top two figures, distal/delayed in bottom two) is plotted at the $(0,0)$ coordinates. The gap and delay axes were rescaled to a standardized $0-1$ range; difference in gap is shown along the bottom left axis, difference in delay along the bottom right axis. 


\section{EXPERIMENT 2}

In order to determine whether the distinctiveness of the direct launching was due to its spatiotemporal contiguity, rather than to its causality, we next examined the discriminability of a spatiotemporally contiguous interaction that was less obviously causal. Thus, any observed uniqueness of spatiotemporal contiguity in this new interaction could be safely attributed to its spatiotemporal properties, and not to its causal nature. The discrimination task in this experiment was identical to that used in the previous experiment, except that the approach and departure vectors for the moving objects were changed to create what we deemed a noncausal interaction. The terminal location of the first object and the initial location of the second were identical to those used in Experiment 1. But the first object approached from above rather than from the left, and the second object moved upward upon movement onset rather than to the right. To ensure that the new interaction was, indeed, less consistently causal than the prototypical launch, the participants judged the causality (on a 1-9 scale) of the range of stimuli experienced in Experiments 1 and 2 .

\section{Method}

Participants. A total of 30 introductory psychology students at Southern Illinois University at Carbondale received course credit for their voluntary participation.

Procedure. The participants performed three tasks: the discrimination task of Experiment 1, but with the new stimuli; a causal ratings task for the new stimuli (16 ratings, 1 each for the four delays by four gaps); and a causal ratings task for the stimuli used in Experiment 1 (16 ratings). Task order was appropriately counterbalanced across participants.

\section{Results and Discussion}

All 30 participants successfully completed the tasks, but 1 participant was dropped for not following directions. The mean accuracy on the discrimination task was $70 \%$ correct, ranging from $41 \%$ to $89 \%$ for individual participants. We replicated the analyses of Experiment 1 and used a similar approach for the ratings data.

Discrimination data. The maximum likelihood parameter estimates are shown in Table 1, and the results are shown graphically in Figure 2. The nlme analysis revealed that the contiguous interaction was judged to be distinctly different from the other stimuli, although not as distinctly as in Experiment 1 (however, cross-experiment comparisons may reflect participant differences and, thus, should be treated with caution). The discriminative control by space and time were approximately equal when the contiguous interaction was first, but the gap had more control when the contiguous interaction was second (a replication of Experiment 1 with these new stimuli).

In contrast, the most distal and delayed interaction was frequently confused with the other distal and/or delayed interactions, especially when the comparison animation involved delay differences rather than gap differences. Control by gap was consistently stronger, especially so when the most distal and delayed interaction was the first

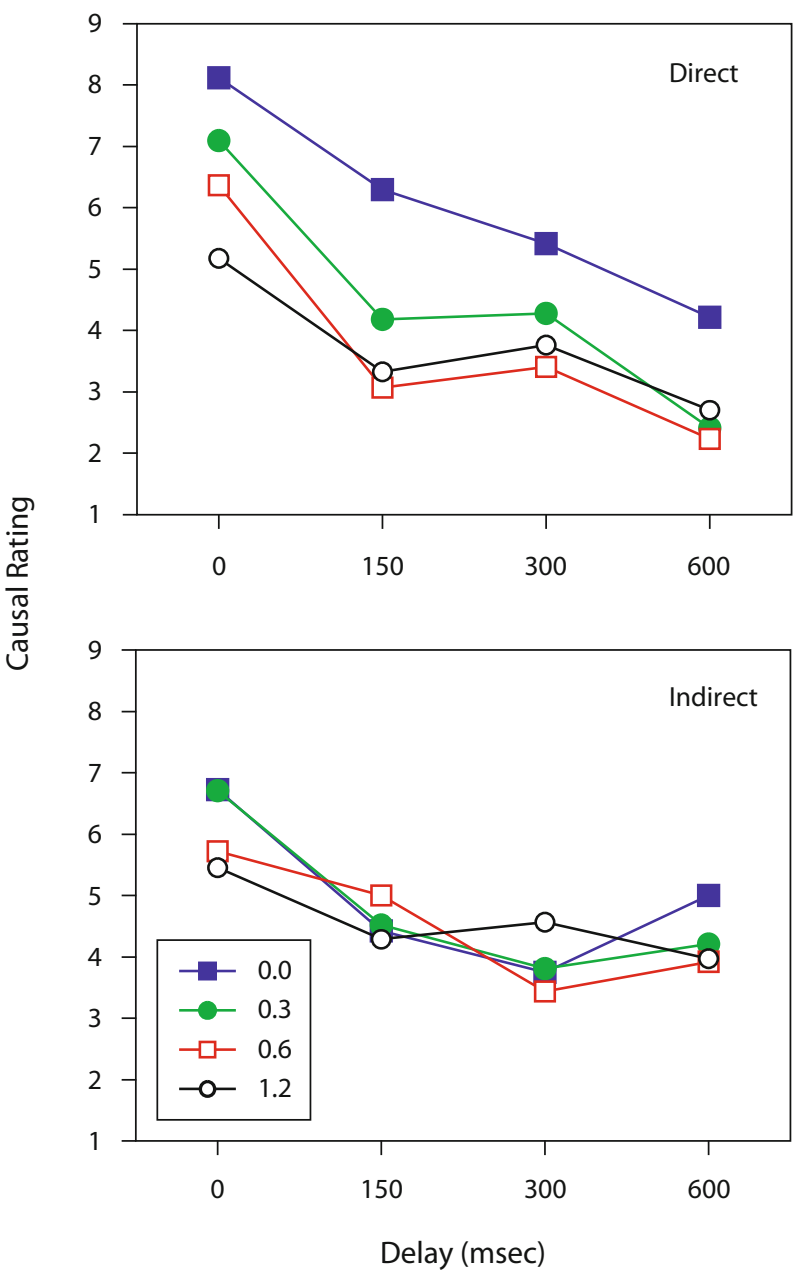

Figure 3. Experiment 2: Causal ratings (1-9 scale) of the interactions as a joint function of delay and gap for the direct approach animations (top) and indirect approach animations (bottom). Unpooled standard errors were consistently in the $[0.4,0.7]$ range.

animation viewed (in this case, control by delay again did not reach statistical significance).

Ratings data. The participants judging the event involving a direct approach (the standard launching paradigm) showed the expected sensitivity to delay and gap differences (see Table 1 and Figure 3), with high ratings for the direct, immediate launch $(M=8.1)$ and increasingly lower ratings for distal and delayed interactions $(M=2.7$ for the $1.2-\mathrm{cm}, 600-\mathrm{msec}$ delay). In contrast, when the event involving the indirect approach was judged, ratings were affected only by the delay (see Table 1), with the contiguous interaction producing a moderate rating $(M=$ 6.7) and the other interactions producing slightly lower ratings (e.g., $M=4.0$ for the $1.2-\mathrm{cm}, 600-\mathrm{msec}$ delay). Any causal distinctiveness for the indirect approach event was driven by the effect of differences in delay, which suggests a natural tendency to impute some degree of causality to events solely on the basis of their temporal contiguity. There were no significant task order effects $(p s>.20)$. 
Thus, although the average ratings were similar across conditions ( $M=4.4$ for direct approach, $M=4.8$ for indirect approach), the indirect approach events produced a profile that suggested significant uncertainty in the form of intermediate ratings for nearly all stimuli and higher participant variability $[S D=3.0$ for the indirect vs. 2.5 for the direct, Welch ANOVA; $t(26)=3.3, p<.01]$. Furthermore, the spatiotemporally contiguous indirect approach event was not as distinctly different from the other indirect approach events. Although the difference was statistically significant using a mixed effects ANOVA [6.7 for no gap and no delay vs. 4.7 for the others; $t(338)=4.6, p<.01$ ], the analogous difference for the direct approach was twice as large $[8.1$ vs. $4.3 ; t(338)=7.4, p<.01]$.

\section{GENERAL DISCUSSION}

The results of Experiment 1 revealed that direct launching was judged to be distinctly different from the other animations in which either a gap or a delay was present. This contrasted with the animation involving the largest gap and delay, which was frequently judged to be identical to those animations involving smaller gaps and/or delays, with errors less likely when the animations differed in the size of the gap rather than in the length of the delay. A similar result was obtained when the interaction was noncausal (Experiment 2), indicating that much of the uniqueness of the direct launch was due to its spatiotemporal contiguity, and not to its causality. This was confirmed by demonstrating that the direct launch received the expected high causal ratings, whereas the spatiotemporally contiguous indirect approach event in Experiment 2 received ratings that were not distinctly different from those for the other events. In addition, the ratings of the noncausal event were exclusively determined by delay, whereas event discriminability was strongly affected by gap and only moderately by delay.

The finding that the direct launch interaction in Experiment 1 appears more distinctive than the indirect contiguous interaction in Experiment 2 may be due to the direct launch's being distinctive in two ways, both spatiotemporally and causally. The fact that spatiotemporal contiguity is distinctive even for noncausal interactions lends credence to our speculation that the concept of causation arose due to the categorical boundary between contiguous and noncontiguous interactions. Unfortunately, the confounding of causality and the extensive experience with this particular instance of spatiotemporal contiguity (launching) makes it difficult to determine how much of the unique character of the launch is due to its spatiotemporal distinctiveness, rather than to its categorical identification as causal.

David Hume (1739/1969) suggested that causality is a psychological construct, a term used to describe particular relations in the world. Although we will not make this stronger claim, it does seem apparent that the most basic and common form of causal relationships, collisions, have spatiotemporal properties that make them distinct from other object interactions that involve action at a distance or delayed action. The emergence of a linguistic label for this unique class of interactions is thus not surprising; there appears to be a categorical distinction between spatiotemporally contiguous interactions and other types of interactions. Michotte's (1946/1963) observation that people's application of the term causal is quite narrow (with very small delays and gaps severely weakening causal impressions) and claims that the launching effect and its variants are perceptually based (Michotte, 1946/1963; Scholl \& Tremoulet, 2000; White, 1988) suggest that these types of causal interactions are strongly influenced by their spatiotemporal properties, although judgments based on these properties may be quite labile and vary across judges (Young et al., 2005).

Collision interactions may have provided the basis of the original concept of causality and, thus, the foundation for the later broadening of the term to other situations. The regularity of these distinctive interactions may lend further credence to their unique nature. We believe that spatiotemporal contiguity signals the possible presence of a causal interaction whose existence can be confirmed either by generalization from other interactions known to possess regularity (distal historical effects) or by extended observation of this particular interaction (proximal historical effects) that either confirms or disconfirms the application of the categorical label of causal. In contrast, the reluctance to label some events as causal even when they possess spatiotemporal contiguity may be a direct function of their unfamiliarity (e.g., the interaction tested in Experiment 2 or the color changes examined by Young \& Falmier, 2008), which can be overcome only after extended observation that confirms the regularity of this interaction.

\section{AUTHOR NOTE}

This research was supported by NSF Grant SES-0720588. Correspondence concerning this article should be addressed to M. E. Young, Department of Psychology, Southern Illinois University, Mailcode 6502, Carbondale, IL 62901-6502 (e-mail: meyoung@siu.edu).

\section{REFERENCES}

Allan, L. G., \& TAngen, J. M. (2005). Judging relationships between events: How do we do it? Canadian Journal of Experimental Psychology, 59, 22-27.

Blakemore, S. J., Fonlupt, P., Pachot-Clouard, M., Darmon, C., Boyer, P., Meltzoff, A. N., et AL. (2001). How the brain perceives causality: An event-related fMRI study. NeuroReport, 12, 37413746.

BueHnER, M. J., \& MAY, J. (2003). Rethinking temporal contiguity and the judgement of causality: Effects of prior knowledge, experience, and reinforcement procedure. Quarterly Journal of Experimental Psychology, 56A, 865-890.

Castro, L., \& Wasserman, E. A. (2007). Discrimination blocking: Acquisition versus performance deficits in human contingency learning. Learning \& Behavior, 35, 149-162.

Fugelsang, J. A., \& Thompson, V. A. (2003). A dual-process model of belief and evidence interactions in causal reasoning. Memory \& Cognition, 31, 800-815.

Gopnik, A., Glymour, C., Sobel, D. M., Schulz, L. E., Kushnir, T., \& DANKs, D. (2004). A theory of causal learning in children: Causal maps and Bayes nets. Psychological Review, 111, 3-32.

Guski, R., \& Troje, N. F. (2003). Audiovisual phenomenal causality. Perception \& Psychophysics, 65, 789-800. 
Hume, D. (1969). A treatise of human nature. New York: Penguin. (Original work published 1739)

Lagnado, D. A., \& Sloman, S. A. (2006). Time as a guide to cause. Journal of Experimental Psychology: Learning, Memory, \& Cognition, 32, 451-460.

LesLIE, A. M. (1984). Spatiotemporal continuity and the perception of causality in infants. Perception, 13, 287-305.

Leslie, A. M., \& Keeble, S. (1987). Do six-month-old infants perceive causality? Cognition, 25, 265-288.

Michotтe, A. (1963). The perception of causality (T. R. Miles \& E. Miles, Trans.). London: Methuen. (Original work published 1946)

Pearl, J. (2000). Causality: Models, reasoning, and inference. Cambridge: Cambridge University Press.

Perales, J. C., Catena, A., Shanks, D. R., \& Gonzalez, J. A. (2005). Dissociation between judgments and outcome-expectancy measures in covariation learning: A signal detection theory approach. Journal of Experimental Psychology: Learning, Memory, \& Cognition, 31, 1105-1120.

Pinheiro, J. C., \& Bates, D. M. (2004). Mixed-effects models in S and $S$-PLUS. New York: Springer.

Rochat, P., Striano, T., \& Morgan, R. (2004). Who is doing what to whom? Young infants' developing sense of social causality in animated displays. Perception, 33, 355-369.
Schlottmann, A., Allen, D., Linderoth, C., \& Hesketh, S. (2002). Perceptual causality in children. Child Development, 73, 1656-1677.

Scholl, B. J., \& NaKayama, K. (2002). Causal capture: Contextual effects on the perception of collision events. Psychological Science, 13, 493-498.

Scholl, B. J., \& Tremoulet, P. (2000). Perceptual causality and animacy. Trends in Cognitive Sciences, 4, 299-309.

Tenenbaum, J. B., Griffiths, T. L., \& Kemp, C. (2006). Theory-based Bayesian models of inductive learning and reasoning. Trends in Cognitive Sciences, 10, 309-318.

Waldmann, M. R., \& HagmaYer, Y. (2005). Seeing versus doing: Two modes of accessing causal knowledge. Journal of Experimental Psychology: Learning, Memory, \& Cognition, 31, 216-227.

White, P. A. (1988). Causal processing: Origins and development. Psychological Bulletin, 104, 36-52.

Young, M. E., \& FAlmier, O. (2008). Color change as a causal agent: Revisited. American Journal of Psychology, 121, 129-157.

Young, M. E., Rogers, E. T., \& Beckmann, J. S. (2005). Causal impressions: Predicting when, not just whether. Memory \& Cognition, 33, 320-331.

(Manuscript received June 16, 2008; revision accepted for publication March 29, 2009.) 